\title{
The role of neutrophil-to-lymphocyte ratio as an outcome predictor factor in acute appendicitis: a single medical center's experience
}

\author{
Bianca L. Grigorescu ${ }^{1}$, Bogdan Andrei Suciu², Irina Saplacan ${ }^{3}$, Raluca S. \\ Fodor ${ }^{3}$, Septimiu Voidazan ${ }^{4}$, Florentina C. Scarlat ${ }^{2}$, Calin Molnar $^{5}$, Ioana \\ Halmaciu $^{2}$ \\ 1. Department of Pathophysiology, George Emil Palade University of Medicine, Pharmacy, Science, \\ and Technology, Romania \\ 2. Department of Anatomy, George Emil Palade University of Medicine, Pharmacy, Science, and \\ Technology of Târgu Mures, Romania \\ 3. Department of Anesthesiology and Intensive Care, Emergency County Hospital, Romania \\ 4. Department of Epidemiology, George Emil Palade University of Medicine, Pharmacy, Science, and \\ Technology, Romania \\ 5. Department of Surgery, George Emil Palade University of Medicine, Pharmacy, Science, and \\ Technology, Romania
}

\begin{abstract}
Introduction: Acute abdominal pain persists in defying modern diagnostic procedures. Inflammatory response variables in urine and blood tests can help rule out other disorders and support a clinical diagnosis of acute appendicitis (AA), but there is no specific blood test that can diagnose AA. In order to address this, our study sought to establish the value of (NLR) as a prognostic and severity predictor in patients with AA. Material and method: We conducted an observational retrospective study of 356 patients who had undergone surgery for AA, assessing $N L R$ as an outcome metric. The NLR was estimated using the differential WBC (white blood count) reported on admission; the data obtained were then recorded in a database and statistically analyzed. Results: The NLR medians correlated $(p<0.001)$ in uncomplicated AA, AA with localized peritonitis, and AA with generalized peritonitis (Kruskal-Wallis test). The ROC curve identified $81.4 \%$ sensitivity, $62.5 \%$ specificity, and a $73 \%$ AUC in localized peritonitis. The NLR value was associated with generalized peritonitis with a sensitivity of $63.7 \%$, specificity of $64.3 \%$, and AUC of $68.2 \%$. The NLR-patient death association had 100\% sensitivity, $80.3 \%$ specificity, and 93.5 $A U C$.
\end{abstract}

* Corresponding author: Bogdan Andrei Suciu, Department of Anatomy, George Emil Palade University of Medicine, Pharmacy, Science, and Technology of Târgu Mures, Romania. E-mail: suciubogdanandrei@yahoo.com 
Conclusion: NLR determination in individuals with acute appendicitis may be useful in predicting complications. $N L R$ values greater than 7.86 are usually related with generalized peritonitis, while values larger than 12.9 may predict an increased risk of patient death. Calculating NLR from a complete blood count is a straightforward and cost-effective method of analyzing complicated AA in resource-constrained settings.

Keywords: appendicitis, inflammation, neutrophil-to-lymphocyte ratio

Received: $2^{\text {nd }}$ October 2021; Accepted: 14 ${ }^{\text {th }}$ December 2021; Published: $17^{\text {th }}$ December 2021

\section{Introduction}

The most common cause of abdominal pain that necessitates surgery is acute appendicitis (AA). In fact, appendectomy is the most common type of emergency surgery performed worldwide. AA affects about $7 \%$ of the population at some point in their lives (1).

Although AA is a reasonably common condition, it can have an unusual onset and progression in certain populations, such as the elderly, children, or pregnant women. Despite multiple modern diagnostic methods, acute abdominal pain continues to present a challenge to rapid and accurate diagnosis, as there are currently no reliable methods of identifying acute appendicitis patients at risk for poor outcomes. The diagnosis of AA cannot be made with one specific test, but judicious use of simple urine and blood tests with particular attention to inflammatory response variables can allow for the exclusion of other illnesses and offer additional evidence to support a clinical diagnosis of appendicitis (2, 3). Appendicitis is often difficult to diagnose not only due to the wide range of symptoms it may induce, but also due to the possibility that the appendix's anatomical position is aberrant. Identifying patients at risk of appendicular perforation as soon as feasible, is critical in clinical practice, as this condition necessitates urgent treatment in order to avoid morbidity and death (4). Abdominal sepsis, also known as secondary peritonitis, is a severe consequence of acute appendicitis caused by gastrointestinal disruption that may result in either localized or diffuse inflammation of the peritoneum and subsequent sepsis (5). A delay in diagnosis could result in peritonitis and abdominal sepsis, both of which are potentially fatal. Thus, diagnosing appendicitis remains difficult since clinical indications or positive blood results can be absent in up to $55 \%$ of patients. Symptoms often overlap with other disorders and the clinical presentation can be atypical, making diagnosis challenging (6).

In the past, a negative appendectomy was used to reduce the morbidity and mortality associated with perforation prior to surgery. As surgery itself is a cause of morbidity and mortality, however, this practice has been considered unacceptable in recent years. AA is an inflammatory pathology whose onset can mimic other diseases with systemic or abdominal localization and unpredictable evolution (7). This emphasizes the significance of neutrophil-to-lymphocyte ratio NLR as a predictive factor. NLR is a helpful tool that is simple to perform as a first test and can be determined at the patient's bedside. The NLR value correlated with the clinical symptomatology can also guide our diagnosis $(7,8)$.

The aim of the present study was to identify the importance of NLR as a prognostic indicator in patients with AA, and as a severity factor in patients with complicated appendicitis.

\section{Material and method}

We conducted a retrospective observational study of 356 patients who underwent surgery for acute appendicitis between January 1, 2017, and May 31, 2021, at the Târgu Mureș County 
Emergency Clinical Hospital in Romania. The study was conducted with permission from the Ethics Commission for Scientific Research (No: 13357/20.05.2021).

For each patient, we gathered demographic information, time of surgery, laboratory test results, and imaging studies. These data were collected from clinical medical records and from the hospital's Pathological Anatomy service records.

Exclusion criteria were: patients with appendicular tumors; patients who had been diagnosed with acute appendicitis during other abdominal procedures; patients with other inflammatory diseases that could have influenced the results of this study.

All patients were diagnosed with acute appendicitis based on clinical examination, blood count, and abdominal ultrasound or abdominal CT. The hospital protocol recommends that abdominal ultrasound be performed as a first step in the imaging diagnosis, and that only patients with clinical and laboratory signs of acute appendicitis but a negative ultrasound examination should undergo an abdominal CT examination.

All subjects in the study underwent complete blood tests (blood count, biochemical examination, and coagulation tests). NLR was considered as the outcome measure in this study. NLR was calculated as ratio of neutrophils to lymphocyte using the differential WBC on admission. The reference values for neutrophils were 1.4-6.5 $* 10^{3} / \mu \mathrm{L}$, and for lymphocytes $1.2-3.4 * 10^{3} / \mu \mathrm{L}$. The absolute number of neutrophils and lymphocytes in peripheral blood are consistent with laboratory reference values and correspond to the age range of 16-99 years (9).

\section{Statistical analysis}

Statistical analysis was performed using SPSS for Windows version 22.0 (SPSS, Inc., Chicago, IL). The associations of NLR with category variables were assessed using Chi-square tests, while differences in continuous variables were analyzed using t Student, Mann Whitney and Kruskal-Wallis tests. We performed ROC curve analysis using MedCalc 13.0 software and calculated the standard error and an exact $\mathrm{Bi}$ nomial Confidence Interval for the Area Under the Curve (AUC). We also calculated associated sensitivity and specificity for all possible threshold values of NLR and determined the optimal criterion values for NLR cut-offs for localized peritonitis, for AA with generalized peritonitis, and for deceased patients. A ROC curve analysis was then used to test the predictive power and to determine the cut-off values of NLR. All tests were two-tailed tests.

\section{Results}

Of the total of 356 patients included in the study, we excluded 13 patients based on the criteria presented above, so our final sample included the medical records of 343 patients. The patients included in the study were aged between 18 and 96 years, with an average female age of 43.3 \pm 19.4 and an average male age of $42.7 \pm 16.5$ years (Table 1).

The recorded mortality was $4.08 \%$ of cases (14 patients).

The distribution of independent values (neutrophils, lymphocytes, NLR) depending on the presence of localized peritonitis, generalized peritonitis, and depending recorded mortality are presented in Table 2.

NLR median value was higher in patients with AA with generalized peritonitis (9.98) than in patients with AA with localized peritonitis (9.22) and significantly higher than in patients with uncomplicated AA (6.21).

We compared the NLR medians among uncomplicated AA, AA with localized peritonitis, and AA with generalized peritonitis using the nonparametric Kruskal-Wallis test and found a statistically significant relation $(\mathrm{p}<0.001)$.

Results of ROC curve analyses for the following 
Table 1. Clinical data of patients included in the study

\begin{tabular}{llc}
\hline Parameter & & Results \\
\hline Sex of patient & Female & 155 patients $(45.19 \%)$ \\
& Male & 188 patients $(54.81 \%)$ \\
\hline Clinical type of appendicitis, diag- & Catarrhal appendicitis & 87 patients $(24.48 \%)$ \\
nosed on the basis of intraoperative & Phlegmonous appendicitis & 104 patients $(30.32 \%)$ \\
appearance & Gangrenous appendicitis & 152 patients $(44.31 \%)$ \\
\hline Histopathological diagnosis & Phlegmonous appendicitis & 168 patients $(48.97 \%)$ \\
& Gangrenous appendicitis & 101 patients $(29.44 \%)$ \\
& Superficial appendicitis & 70 patients $(20.40 \%)$ \\
& Normal histological appendix & 4 patients $(1.16 \%)$ \\
\hline Abdominal complications of acute ap- & Localized peritonitis & 28 patients $(8.16 \%)$ \\
pendicitis & Generalized peritonitis & 68 patients $(19.82 \%)$ \\
\hline
\end{tabular}

Table 2. Distribution of independent values of lymphocytes, neutrophils and NLR in association with localized peritonitis, generalized peritonitis, and recorded mortality

\begin{tabular}{|c|c|c|c|c|c|c|c|}
\hline & $\begin{array}{l}\text { Lym } \\
\pm \text { sta }\end{array}$ & $\begin{array}{l}\text { ocytes, mean } \\
\text { ard deviation }\end{array}$ & $\begin{array}{c}P \\
\text { value }\end{array}$ & $\begin{array}{l}\text { Neutrophils, mean } \pm \\
\text { standard deviation }\end{array}$ & $\begin{array}{c}P \\
\text { value }\end{array}$ & $\begin{array}{l}\text { NLR, median } \\
\text { (min-max) }\end{array}$ & $\begin{array}{c}P \\
\text { value }\end{array}$ \\
\hline \multirow{2}{*}{$\begin{array}{l}\text { Localized } \\
\text { peritonitis }\end{array}$} & Yes & $1.62 \pm 0.81$ & \multirow{2}{*}{0.003} & $12.17 \pm 4.23$ & \multirow{2}{*}{0.0001} & $9.22(3.29-40.38)$ & \multirow{2}{*}{0.02} \\
\hline & No & $2.16 \pm 0.93$ & & $9.18 \pm 4.49$ & & $6.76(0.25-72.18)$ & \\
\hline \multirow{2}{*}{$\begin{array}{l}\text { Generalized } \\
\text { peritonitis }\end{array}$} & Yes & $1.51 \pm 0.79$ & \multirow{2}{*}{0.03} & $12.82 \pm 4.23$ & \multirow{2}{*}{0.0005} & $9.98(2.09-72.18)$ & \multirow{2}{*}{0.0001} \\
\hline & No & $1.88 \pm 0.89$ & & $10.7 \pm 4.47$ & & $6.43(0.2-40.38)$ & \\
\hline \multirow{2}{*}{ Deaths } & Yes & $1.62 \pm 0.81$ & \multirow{2}{*}{0.03} & $12.17 \pm 4.23$ & \multirow{2}{*}{0.01} & $40.38(12.9-72.18)$ & \multirow{2}{*}{0.009} \\
\hline & No & $2.16 \pm 0.93$ & & $9.18 \pm 4.49$ & & $6.80(0.2-31.5)$ & \\
\hline
\end{tabular}

comparisons: 1) NLR value and the presence of localized peritonitis; 2) NLR value and the presence of generalized peritonitis 3) NLR value and patient death.

In cases of localized peritonitis, we obtained a cutoff value of 4.29 for NLR and the ROC curve and detected a sensitivity of $81.4 \%$, a specificity of $62.5 \%$. The value of the area under the curve (AUC) was 0.73 with a $95 \%$ confidence interval (Figure 1).

In cases of generalized peritonitis, we had a NLR cutoff value of 7.86 , a sensitivity of $63.7 \%$, and a specificity of $64.3 \%$. The AUC was 0.682 with a $95 \%$ confidence interval (Figure 2 ).

When testing the association between NLR and patient death, we determined a cutoff value of NLR of 12.9 , with a sensitivity of $100 \%$ and a specificity of $80.3 \%$. The AUC was 0.935 with a 95\% confidence interval (Figure 3 ).

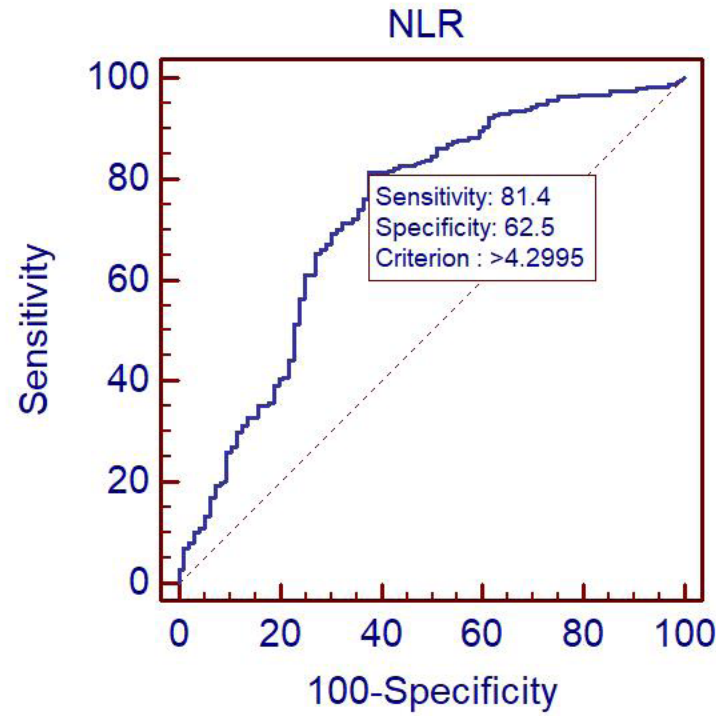

Fig. 1. ROC curve for the association between NLR value and the existence of localized peritonitis. 


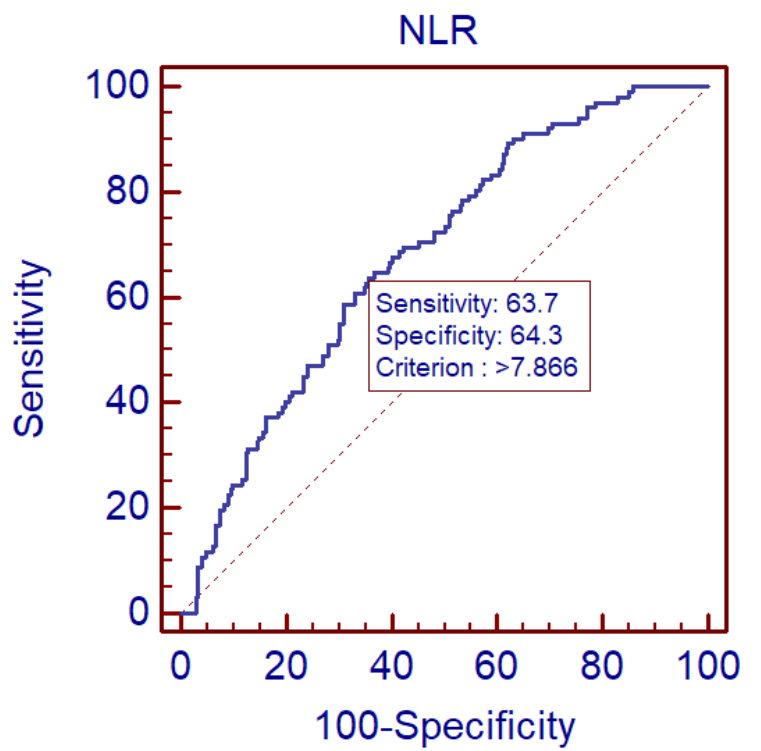

Fig. 2. ROC curve for the association between

NLR value and the existence of generalized peritonitis.

\section{Discussion}

Despite significant improvements in the diagnosis of AA, mainly thanks to imaging techniques, several diagnostic hazards remain, and they may lead to a large number of misdiagnoses and/or needless surgery. Appendicitis is most common between the ages of 10 and 20, but no age group is exempt. Most demographic studies show a slight male predominance, with an overall male: female ratio of 1.4:1. In the United States, the overall lifetime risk is 8.6 percent for males and 6.7 percent for females (3). Such male predominance was confirmed also in the present study. Some authors suggest that neutrophil-to-lymphocyte ratio (NLR) is a reliable predictor of inflammation and is therefore useful in the preoperative diagnosis of AA (7), but the results of our study emphasize the NLR prognostic value rather than its diagnostic significance.

Out of all cases of acute appendicitis, the evolution towards perforation varies in the literature between $16 \%$ and $40 \%$, with the incidence of

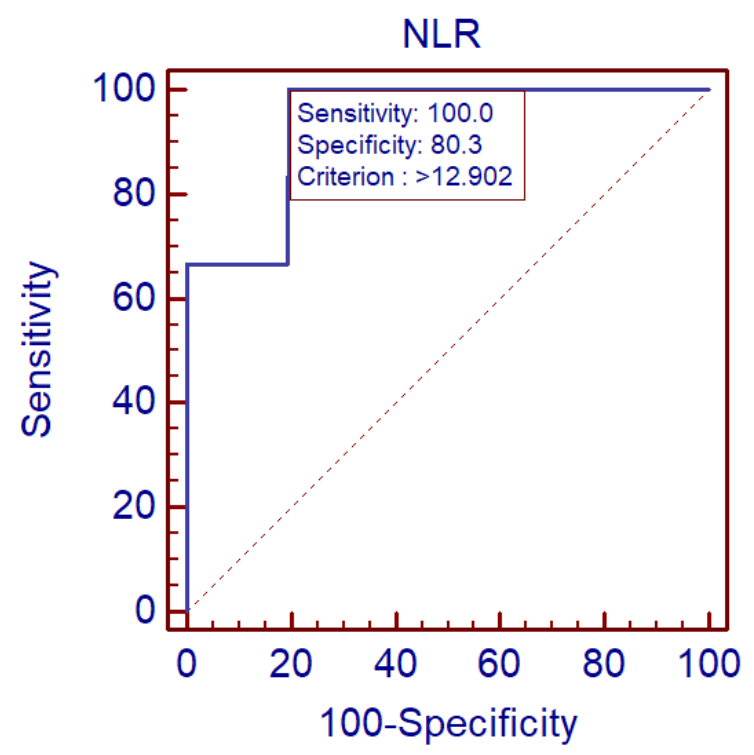

Fig. 3. ROC curve for the association between NLR value and patient death.

appendicular perforation being higher in young people and the elderly (10). Patients with acute perforated appendix and extensive peritonitis have a $5 \%$ mortality rate (11). In our study, one third of patients had a complicated form of appendicitis, of which $8.16 \%$ had localized peritonitis and $19.82 \%$ had generalized peritonitis. Our study recorded a mortality of $4.08 \%$, of which all deaths were due to septic shock or its complications.

In recent years, the use of emergency abdominal CT scans in patients with suspected acute appendicitis has become mandatory. Studies have indicated that this practice has reduced the number of appendectomy cases performed on patients with a normal histological appendix, an effect observed in children and adults alike $(12,13)$. Although abdominal CT has revealed greater specificity and sensitivity than abdominal ultrasonography in detecting acute appendicitis (13), some authors caution against broad use of abdominal CT scans, especially in children, due to the danger of developing neoplasms from radia- 
tion exposure over the long term (14). Accordingly, our study found that abdominal ultrasound was performed as a first step in the imaging diagnosis, while CT examination was performed as a last resort, and only in cases of high suspicion of AA (based on clinical and laboratory findings) with negative ultrasound. Still, a small percentage $(1.16 \%)$ of patients were determined to have a normal histological appendix.

Given the risks of negative appendectomy in patients with clinical suspicion of acute appendicitis, and the fact that it is a common condition that can be treated surgically with low morbidity and low mortality if performed correctly, any diagnostic test that can identify the underlying cause of appendicitis should be considered a life-saving tool. In clinical practice, it is common to encounter suspected cases of acute appendicitis in which abdominal CT is contraindicated, such as in the case of pregnant women. The utilization of less invasive diagnostic techniques could therefore be advantageous for patients in certain circumstances (15).

Although multiple serological diagnostic markers, such as interleukin 6 , interleukin 8 , and C-reactive protein, have been tested in patients with clinical suspicion of acute appendicitis, none of these markers have been shown to be useful, especially in the early stages of acute appendicitis (16). A major advantage of NLR determination is that blood tests are routine and low-cost (17), and inflammatory subclinical markers such as NLR can be easily calculated from differential WBC. Furthermore, NLR may also predict appendicitis severity by providing information on two different immune and inflammatory pathways $(7,15)$. NLR is regularly used as an indirect measure of systemic inflammatory syndrome in many pathologies. The level of neutrophils in the blood is a reliable indicator of local inflammation, while the level of lymphocytes in the blood is a reliable indicator of the inflammatory syndrome's regulatory pathways. Because the ratio is also elevated in other inflammatory diseases, NLR as a diagnostic parameter has little specificity for AA and may be a source of false-positive error. This led us to rule out other inflammatory diseases as exclusion factors in our study.

Many studies have been conducted to determine whether or not NLR plays a clinically significant role in appendicitis $(15,18)$. Our study revealed a significant positive correlation between NLR in uncomplicated AA and complicated AA ( $p$ $<0.001)$. Given these findings, we can conclude that NLR is a strong predictor of the occurrence of critical septic conditions as well as death in patients with acute appendicitis. These findings are consistent with other studies that have claimed that the NLR can predict mortality and antibiotic response in ICU patients with sepsis and septic shock (19-21).

Delayed diagnosis or misdiagnosis of AA can increase the risk of perforation and abdominal sepsis. According to our research, the chance of developing severe complications, including generalized peritonitis and death, increases exponentially as the NLR value increases. NLR increased exponentially in patients with complicated AA compared to patients with uncomplicated AA, and the NLR value in patients with localized peritonitis was lower than in patients with generalized peritonitis. In cases of mortality, the mean value of NLR exceeded that of patients with generalized peritonitis. These aspects have been observed previously by researchers who have noted that NLR has a better sensitivity and specificity than other serological markers in predicting the incidence of complications in patients with acute appendicitis (22-24).

The present study has some limitations. Despite our large sample size, the total incidence of acute appendicitis necessitates further study. Additionally, the fact that the study is based on the experience of a single medical center may skew the results in unpredictable ways. 


\section{Conclusion}

Determination of NLR in patients with acute appendicitis may be of particular clinical importance, especially in predicting complications. NLR values above 7.86 are frequently associated with generalized peritonitis, and NLR values greater than 12.9 may present an additional risk for patient death. The calculation of NLR from a full blood count is a simple, cost-effective, and readily available metric for assessing complicated/perforated AA in resource-limited situations.

\section{Abbreviations}

AA- acute appendicitis

NLR - neutrophil-to-lymphocyte ratio

AUC- Area Under the Curve

WBC - white blood count

\section{Author Contributions}

Conceptualization, B-L.G.; methodology B-L.G and C.M.; software S.V.; validation B.A.S. and I.H; formal analysis S.V. and I.S.; investigation C.F.S. and R.S.F.; resources C.F.S. and R.S.F. ; data curation S.V.; writing - original draft preparation B-L.G. and C.M.; writing-review and editing B.A.S. and I.H; visualization B.L.G and B.A.S.; supervision, I.H.; project administration B.L.G, I.S. and I.H. All authors have read and agreed to the published version of the manuscript.

\section{Conflicts of Interest}

The authors declare no conflict of interest.

\section{References}

1. Bhangu A, Søreide K, Di Saverio S, Assarsson JH, Drake FT. Acute appendicitis: modern understanding of pathogenesis, diagnosis, and management. Lancet. 2015 Sep 26;386(10000):1278-87. DOI: $10.1016 /$ S0140-6736(15)00275-5

2. Cox J, Sovak G. Missed appendicitis diagnosis: A case report. J Can Chiropr Assoc. 2015 Sep;59(3):294-9.

3. Humes DJ, Simpson J. Acute appendicitis. BMJ. 2006 Sep 9;333(7567):530-4. DOI: 10.1136/ bmj.38940.664363.AE

4. Shogilev DJ, Duus N, Odom SR, Shapiro NI. Diagnosing appendicitis: evidence-based review of the diagnostic approach in 2014. West J Emerg Med. 2014 Nov;15(7):859-71. DOI: 10.5811/westjem.2014.9.21568

5. Boldingh QJJ, de Vries FEE, Boermeester MA. Abdominal sepsis. Curr Opin Crit Care. 2017 Apr;23(2):15966. DOI: 10.1097/MCC.0000000000000388

6. Kabir SA, Kabir SI, Sun R, Jafferbhoy S, Karim A. How to diagnose an acutely inflamed appendix; a systematic review of the latest evidence. Int J Surg. 2017 Apr;40:155-62. DOI: 10.1016/j.ijsu.2017.03.013

7. Kahramanca S, Ozgehan G, Seker D, Gökce EI, Seker G, Tunç G, et al. Neutrophil-to-lymphocyte ratio as a predictor of acute appendicitis. Ulus Travma Acil Cerrahi Derg. 2014 Jan;20(1):19-22. DOI: 10.5505/ tjtes.2014.20688

8. Schellekens DHSM, Hulsewé KWE, van Acker BAC, van Bijnen AA, de Jaegere TMH, Sastrowijoto SH, et al. Evaluation of the diagnostic accuracy of plasma markers for early diagnosis in patients suspected for acute appendicitis. Acad Emerg Med. 2013 Jul;20(7):703-10. DOI: 10.1111/acem.12160

9. Rodak, Bernadette F, George A. Fritsma and Elaine M. Keohane, Hematology/Hemostasis Reference Intervals Elsevier Saunders, Hematology: Clinical Principles and Applications. St. Louis, Mo.:, 2016. ISBN 978-0-32323

10. Livingston EH, Woodward WA, Sarosi GA, Haley RW. Disconnect between incidence of nonperforated and perforated appendicitis: implications for pathophysiology and management. Ann Surg. 2007 Jun;245(6):88692. DOI: 10.1097/01.sla.0000256391.05233.aa

11. Acute appendicitis--appendectomy or the "antibiotics first” strategy. N Engl J Med. 2015 Jun 4;372(23):2274. DOI: $10.1056 /$ NEJMx150021

12. Krajewski S, Brown J, Phang PT, Raval M, Brown CJ. Impact of computed tomography of the abdomen on clinical outcomes in patients with acute right lower quadrant pain: a meta-analysis. Can J Surg. 2011 Feb;54(1):43-53. DOI: 10.1503/cjs.023509

13. Carroll PJ, Gibson D, El-Faedy O, Dunne C, Coffey C, Hannigan A, et al. Surgeon-performed ultrasound at the bedside for the detection of appendicitis and gallstones: systematic review and meta-analysis. Am J Surg. 2013 Jan;205(1):102-8. DOI: 10.1016/j.amjsurg.2012.02.017

14. Smith-Bindman R, Lipson J, Marcus R, Kim KP, Mahesh $\mathrm{M}$, Gould R, et al. Radiation dose associated with common computed tomography examinations and the associated lifetime attributable risk of cancer. Arch Intern Med. 2009 Dec 14;169(22):2078-86. DOI: 
10.1001/archinternmed.2009.427

15. Hajibandeh S, Hajibandeh S, Hobbs N, Mansour M. Neutrophil-to-lymphocyte ratio predicts acute appendicitis and distinguishes between complicated and uncomplicated appendicitis: A systematic review and meta-analysis. Am J Surg. 2020 Jan;219(1):154-63. DOI: 10.1016/j.amjsurg.2019.04.018

16. Käser SA, Fankhauser G, Willi N, Maurer CA. C-reactive protein is superior to bilirubin for anticipation of perforation in acute appendicitis. Scand J Gastroenterol. 2010 Aug;45(7-8):885-92. DOI: $10.3109 / 00365521003728572$

17. Alvarado A. Inflammatory Markers in Acute Appendicitis: Are we Still Looking for the Philosopher's Stone? Journal of Surgery. 2018;2:1-5. DOI: 10.29011/25759760.001104

18. Acarturk G, Acay A, Demir K, Ulu MS, Ahsen A, Yuksel S. Neutrophil-to-lymphocyte ratio in inflammatory bowel disease - as a new predictor of disease severity. Bratisl Lek Listy. 2015;116(4):213-7. DOI: 10.4149/ BLL_2015_041

19. Sarı $\bar{R}$, Karakurt Z, Ay M, Çelik ME, Yalaz Tekan Ü, Çiyiltepe F, et al. Neutrophil to lymphocyte ratio as a predictor of treatment response and mortality in septic shock patients in the intensive care unit. Turk J Med Sci. 2019 Oct 24;49(5):1336-49. DOI: 10.3906/sag1901-105

20. Hwang SY, Shin TG, Jo IJ, Jeon K, Suh GY, Lee
TR, et al. Neutrophil-to-lymphocyte ratio as a prognostic marker in critically-ill septic patients. Am J Emerg Med. 2017 Feb;35(2):234-9. DOI: 10.1016/j. ajem.2016.10.055

21. Marjanovic V, Budic I, Jankovic-Velickovic L, Stevic M, Kostic M, Simic D. Predictive Value of Neutrophil Count for Postoperative Complications in Children after Surgery of Perforated Appendicitis. Rev Romana Med Lab. 2021;29(1):77-84. DOI: 10.2478/rrlm-20210008

22. Delgado-Miguel C, Mu-oz-Serrano AJ, Barrena Delfa S, Nú-ez Cerezo V, Estefanía K, Velayos M, et al. [Neutrophil-to-lymphocyte ratio as a predictor of peritonitis in acute appendicitis in children]. Cir Pediatr. 2019 Oct $1 ; 32(4): 185-9$.

23. Ahmad KA, Ideris N, Aziz SHSA. A Cross-Sectional Study of Neutrophil-to-Lymphocyte Ratio in Diagnosing Acute Appendicitis in Hospital Melaka. Malays J Med Sci. 2019 Nov;26(6):55-66. DOI: 10.21315/ mjms2019.26.6.6

24. Molnar C, Nicolescu CL, Botoncea M, Butiurca VO, Suciu BA, Hălmaciu I, et al. The predictive role of platelet to lymphocyte ratio in the occurrence of anastomotic complications following gastric resections for neoplasia - single centre experience. Rev Romana Med Lab. 2020 Mar 10;28(2):185-94. DOI: 10.2478/rrlm2020-0011 